Conclusions EOS in term infants is associated with significantly lower maternal and neonatal 25-OHD levels. We also found that levels of $25-\mathrm{OHD}$ in neonates were positively correlated with those in mothers. These data suggest that adequate vitamin D supplementation during pregnancy may be helpful to prevent EOS in term neonates.

\section{PS-222 STAPHYLOCOCCUS CAPITIS IN NEONATAL LATE-ONSET SEPSIS: UNEXPECTED WORLDWIDE DISSEMINATION OF AN ENDEMIC MULTI-RESISTANT CLONE}

${ }^{1} \mathrm{M}$ Butin, ${ }^{2} \mathrm{P}$ Martins Simoes, ${ }^{3} \mathrm{H}$ Lemriss, ${ }^{3} \mathrm{~S}$ Lemriss, ${ }^{2} \mathrm{~F}$ Vandenesch, ${ }^{4} \mathrm{O}$ Claris, ${ }^{4} \mathrm{JC}$ Picaud, ${ }^{1} \mathrm{JP}$ Rasigade, ${ }^{1} \mathrm{~F}$ Laurent. ${ }^{1}$ Clinical Microbiology, Hospices Civils de Lyon, Lyon, France; ${ }^{2}$ U1111 - CIRI, Inserm, Lyon, France; ' ${ }^{2}$ Laboratoire de Recherche Et d'Analyses Médicales, Fraternelle de La Gendarmerie Royale, Rabbat, Morocco; ${ }^{4}$ NICU, Hospices Civils de Lyon, Lyon, France

\subsection{6/archdischild-2014-307384.521}

Background Multi-resistant Staphylococcus capitis NRCS-A is involved in late-onset sepsis (LOS) in French NICUs.

Aims To investigate the geographical distribution of NRCS-A, and to precise its susceptibility profile.

Methods Twelve S. capitis isolates from distant NICUs (Australia, Belgium, France, United Kingdom, $\mathrm{n}=3$ each) and $2 S$. capitis isolates from adult patients were analysed using PFGE, SCCmec typing, dru-typing, a MLST-like analysis, and antimicrobial susceptibility testing. To explore impact of vancomycin selective pressure, after 15 daily subcultures with vancomycin, we determined vancomycin, daptomycin and linezolid minimal inhibitory concentrations (MICs).

Results All NICU S. capitis (i) shared $>80 \%$ similarity of PFGE profile and were similar to NRCS-A profile, (ii) harboured a type V-related SCCmec element, (iii) exhibited the same drutype, (iv) formed a monophyletic group, (v) harboured a same antimicrobial susceptibility profile including aminoglycosides and methicillin resistance, and vancomycin heteroresistance. These molecular and antimicrobial susceptibility profiles differed from those of adult isolates. An increase of vancomycin and daptomycin (but not linezolid) MICs was observed, significantly faster $(\mathrm{p}<0.05)$ for NRCS-A isolates than other tested strains.

Conclusion Our analysis demonstrates an unexpected worldwide distribution of $S$. capitis NRCS-A, specifically in NICUs. Recently, we collected complementary NICU isolates belonging to NRCS-A from Norway, Denmark, the Netherlands, USA, Brazil, New Zealand and Canada, confirming the worrisome dissemination of NRCS-A. Its multi-resistant profile and its ability to rapidly adapt to vancomycin selective pressure, constitute a selective advantage to NRCS-A in NICUs, and raise the issue of potential therapeutic failure and the need for alternative antimicrobial regimens.

\section{Neonatology Clinical}

\section{PS-223 IMPROVING STAFF COMPETENCE AND CONFIDENCE FOR NEONATAL RESUSCIATION, A QUALITY IMPROVEMENT INITIATIVE}

S Hackett, M Casssidy, S Gormally. Neonatal Intensive Care Unit, Our Lady of Lourdes Hosptial Drogheda Co. Louth, Drogheda, Ireland

10.1136/archdischild-2014-307384.522
Aims To introduce a Quality Improvement Initiative aimed at instilling confidence and competence with Neonatal Resuscitation in Labour Ward (LW) and Neonatal Unit (NICU) staff.

Methods A Neonatal Resuscitation Initiative Team (NRIT) consisted of LW and NICU midwives, an Advanced Nurse Practitioner (ANP) and a Paediatric Consultant. LW, NICU and paediatric staff were invited to attend a presentation to outline the aims and methodology of the initiative. Emphasis was placed on teamwork with effective leadership and communication. This was followed by a demonstration of 2 low fidelity simulated neonatal resuscitations performed by the NRIT.

Midwifery and NICU staff were then invited to partake in a Simulated Team Response (STR) to 2 simulated resuscitations.

A random selection of participants completed a closed questionnaire before and after the NRIT demonstration and before and after their STR. Levels of confidence in all aspects of neonatal resuscitation, including teamwork and leadership skills were evaluated using a Likert Scale.

Results One hundred and thirteen staff participated in the quality initiative from January 2013 to August 2013. Seventy four staff participated in the NRIT demonstrations and 39 in the STR. Forty three questionnaires were completed following the NRIT and 13 following the STR. Using a Wilcoxon test the prepost gain in confidence score was statistically significant $(\mathrm{p}=$ 0.0020) for doctors and midwives.

Conclusion Simulation training is a valuable means of maintaining skills and enhancing confidence for neonatal resuscitation. This study quantifies the positive impact of a Quality Improvement Initiative aimed at improving performance at neonatal resuscitation.

\section{PS-224 VIDEO EDUCATION TO IMPROVE BAG MASK VENTILATION DURING SIMULATED NEWBORN RESUSCITATION}

${ }^{1} \mathrm{P}$ Deindl, ${ }^{2} \mathrm{~S}$ Schwindt, ${ }^{2} \mathrm{~A}$ Berger, ${ }^{3} \mathrm{G}$ Schmoelzer. ${ }^{1}$ Department of Neonatology and Pediatric Intensive Care Medicine, University Medical Center Hamburg-Eppendorf, Hamburg, Germany; ${ }^{2}$ Department of Pediatrics and Adolescent Medicine Division of Neonatology Pediatric Intensive Care and Neuropediatrics, Medical University of Vienna, Vienna, Austria; ${ }^{3}$ Department of Pediatrics and Adolescent Medicine Division of Neonatology Pediatric Intensive Care and Neuropediatrics, Royal Alexandra Hospital, Edmonton, Canada

\subsection{6/archdischild-2014-307384.523}

Aim To evaluate if video based education could improve quality of positive pressure ventilation (PPV) performed by novice health care providers during neonatal resuscitation.

Methods Twenty-eight 4th year medical students were randomly paired and instructed to give PPV to a modified manikin as single-person resuscitators, then as two-person paired resuscitators using either an anatomical shaped neonatal face mask with an air cushion rim (IS) or a Laerdal round face mask (LM). After watching a video-tutorial they randomly repeated each mask ventilation performance. Airway pressure, gas flow, tidal volume, and mask leak were recorded. PPV performance quality was analysed using video recording.

Results Mask leak was lower during one-person ventilation when using IS $(56 \pm 16 \%)$ compared to LM (71 $\pm 19 \%)$. LM mask leak during one-person ventilation was significantly lower when using the two point top hold in contrast to the ok rim hold (before training: $63 \pm 22 \%$ vs. $72 \pm 18 \%$, after training: $57 \pm 17 \%$ vs. $77 \pm 12 \%$, respectively). Watching a video-tutorial improved correct head position (score: $1.4 \pm 0.7$ vs. $3.8 \pm$ 